\title{
Perfil cardiometabólico adverso de pacientes sanos en exámenes clínicos periódicos de salud
}

\author{
Adverse cardiometabolic profile in a healthy adult population during clinical \\ check up
}

Lorena Ivonne Sarati, Javier Vallejos, Daina Schvartzman, Jimena Díaz Carreño, Vanesa Tujsnaider, Yanina Troncoso, Rodolfo Bado, Patricia Carrascosa, Gastón A. Rodríguez-Granillo

\section{RESUMEN}

Objetivo. Reportar la prevalencia de factores de riesgo cardiovasculares, perfil cardiometabólico adverso y de estudios diagnósticos patológicos asociados en una población joven de individuos sanos que concurren a realizarse un examen clínico periódico de salud (ECP) en un centro diagnóstico de referencia de la Ciudad Autónoma de Buenos Aires.

Métodos. Se incluyeron pacientes de ambos sexos sin antecedentes cardiológicos conocidos de entre 18 y 80 años. El ECP incluyó examen clínico, estudios de sangre, electrocardiograma, prueba ergométrica graduada y ecocardiograma Doppler. Resultados. Se incluyeron 311 individuos asintomáticos consecutivos (53\% hombres). La edad media fue de $42,4 \pm 14$ años, y el $48 \%$ presentó sobrepeso u obesidad. El $10 \%$ registró una presión arterial sistólica $\geq 140 \mathrm{mmHg}$ y/o diastólica $\geq 90 \mathrm{mmHg}$ de los cuales el $65 \%$ no tenía antecedentes de hipertensión. El 37\% de los hombres presentó alteraciones del perfil lipídico. Diez (3\%) pacientes presentaron alteraciones electrocardiográficas y/o en la prueba ergométrica, y 46 (15\%) presentaron una capacidad funcional limitada (<7 METS). El 14\% de la población se ubicó dentro de grupo de riesgo elevado de eventos cardiovasculares y el $20 \%$ de los ECP fueron clasificados como anormales, concentrándose la mayor proporción en hombres mayores de 40 años.

Conclusión. Identificamos tasas relativamente elevadas de perfil cardiometabólico adverso en una población asintomática, socialmente activa, con manejo subóptimo de los factores de riesgo coronario.

\begin{abstract}
Objective. To report the prevalence of cardiovascular risk factors, adverse cardiometabolic profile, and abnormal diagnostic tests associated in a young population of healthy individuals self-referred for a clinical checkup (CC) in a tertiary diagnostic facility located in the city of Buenos Aires.

Methods. Patients of both sexes without known cardiac history between 18 and 80 years were included. The CC included physical examination, blood workup, 12-lead electrocardiogram (ECG), exercise ECG, and Doppler echocardiography.

Results. A total of 311 consecutive asymptomatic patients (53\% male) we included. The mean age was $42.4 \pm 14$ years, and $48 \%$ were overweight or obese. Ten percent had a systolic blood pressure $\geq 140 \mathrm{mmHg}$ and/or diastolic $\geq 90 \mathrm{mmHg}$, of which $65 \%$ had no history of hypertension. An impaired lipid profile was identified in 59 (37\%) of the males. Ten (3\%) patients had ECG and/or exercise ECG abnormalities, and 46 (15\%) had limited functional capacity (<7 METS). Fourteen percent of the population was classified at high risk for cardiovascular events, and 20\% of the CC were classified as abnormal, most clustered among men older than 40 years.

Conclusion. We identified relatively high rates of adverse cardiometabolic profile in an asymptomatic population, with sub-optimal management of coronary risk factors.
\end{abstract}

Key words: checkups, risk factors, cardiovascular disease, prevention.

Palabras claves: chequeos, factores de riesgo, enfermedad cardiovascular, prevención.

Revista Argentina de Cardioangiología Intervencionista 2016;7(3):129-135

\section{INTRODUCCIÓN}

A pesar de la reducción marcada en la mortalidad observada en las últimas décadas, la enfermedad cardiovascular continúa siendo la principal causa de muerte a nivel global. ${ }^{1}$ Existe una estrecha asociación entre los factores de riesgo cardiovascular (FRC) y la extensión de la aterosclerosis coronaria y periférica ${ }^{2,3}$. El conocimiento de la prevalencia de FRC es vital desde el punto de vista epi-

1. Departamento de Investigación, Diagnóstico Maipú. Argentina.

$\triangle$ Correspondencia: Dr. Gastón A. Rodríguez Granillo | Av. Maipú 1668, Vicente López, (B1602ABQ) Buenos Aires, Argentina | Tel/fax: +5411 48377596 | grodriguezgranillo@gmail.com demiológico. Aproximadamente la mitad de la reducción de la mortalidad cardiovascular observada en las últimas décadas se atribuyó a la reducción de los FRC, mientras que estrategias terapéuticas avanzadas como la revascularización otorgaron apenas un $7 \%$ de reducción. ${ }^{2}$

Grandes estudios multicéntricos prospectivos han demostrado que la adherencia a un estilo de vida que incluya la abstención de fumar, un índice de masa corpo$\mathrm{ral}$ (IMC) óptimo $\left(<25 \mathrm{~kg} / \mathrm{m}^{2}\right)$, actividad física regular y una dieta mediterránea reducen significativamente la mortalidad $^{4,5}$. De hecho, la adherencia a dicho estilo de vida se asocia a una menor incidencia de calcificación arterial coronaria (CAC) y de su progresión, que constituyen un predictor independiente de mortalidad en seguimientos prolongados de hasta 15 años ${ }^{6,7}$.

Grandes estudios poblacionales como el Cardiovascular Risk in Young Finns y el Coronary Artery Risk De- 
velopment in Young Adults (CARDIA) demostraron que la presencia y niveles de FRC (particularmente del LDL-C y la presión arterial sistólica) durante la adolescencia y juventud favorecen el desarrollo de CAC durante la adultez ${ }^{8}$. Por ejemplo, en el estudio CARDIA los niveles subóptimos de LDL-C y HDL-C durante la adultez temprana fueron predictores independientes de aterosclerosis coronaria dos décadas más tarde, y prácticamente la mitad de los pacientes con algún grado de CAC a los 45 años tenían niveles de LDL-C mayores de $160 \mathrm{mg} / \mathrm{dl}$ veinte años antes?

El objetivo de nuestro estudio fue reportar la prevalencia de FRC, perfil cardiometabólico adverso y estudios diagnósticos asociados patológicos en una población joven de individuos sanos que concurren a realizarse un examen clínico periódico de salud (ECP) en un centro diagnóstico de referencia de la Ciudad Autónoma de Buenos Aires.

\section{MÉTODOS}

Estudio observacional retrospectivo, que consistió en la inclusión durante el mes de marzo 2015 de pacientes consecutivos asintomáticos, que concurrieron por sus propios medios a realizarse un ECP en la sede Cabildo de Diagnóstico Maipú, de la Ciudad Autónoma de Buenos Aires. Se incluyeron pacientes de ambos sexos de entre 18 y 80 años. El estudio de ECP comprendió la confección de una historia clínica a cargo de un médico clínico matriculado donde se constató estilo de vida y la presencia de FRC (actividad física, tipo de alimentación, hábito de fumar, historia de hipertensión arterial, diabetes, dislipemia, antecedentes personales de enfermedades conocidas), medidas antropométricas [peso $(\mathrm{kg})$, talla $(\mathrm{m})$ acordes a las normativas internacionales], registro de la tensión arterial y frecuencia cardíaca de acuerdo a las normativas de la American Heart Association y el consenso Latinoamericano de Hipertensión Arterial. Los exámenes a realizar fueron preestablecidos y determinados de acuerdo al sexo y la edad, e incluyeron: examen de orina completo, una muestra de sangre donde se analizó el hemograma, hepatograma, glucemia, uremia, creatinina, tirotrofina, perfil lipídico [colesterol total, HDL-C, LDL-C (método enzimático colorimétrico), triglicéridos (TG), índice aterogénico (colesterol total/HDL-C) y el cociente TG/HDL-C como marcador de resistencia a la insulina]. Asimismo, se realizó una radiografía de tórax de frente, un electrocardiograma (ECG) de 12 derivaciones y una prueba ergométrica graduada, PEG [protocolo de Bruce (Ergo View Kiprun KR-325)] y ecocardiograma Doppler (VID APLIO 300 Toshiba). En pacientes mayores de 50 años se analizó además el antígeno prostático específico, y se realizó una ecografía abdominal y una consulta oftalmológica, mientras que en los mayores de 65 años se agregaron una audiometría y un eco-Doppler de vasos de cuello.

\section{Definiciones}

Se definió por consenso la presencia o ausencia de ECP normal o anormal, definiéndose como anormal la identificación de alguno de los siguientes: hipertensión (presión arterial sistólica $\geq 140 \mathrm{~mm} / \mathrm{Hg}$ o diastólica $\geq 90 \mathrm{~mm} / \mathrm{Hg}$ ), anemia (definida como hemoglobina $<13 \mathrm{mg} / \mathrm{dl}$ en hombres y $<12 \mathrm{mg} / \mathrm{dl}$ en mujeres), colesterol anormal (definido como LDL-C $>160 \mathrm{mg} / \mathrm{dl}$, o HDL-C $<40 \mathrm{mg} / \mathrm{dl}$, TG $>200 \mathrm{mg} / \mathrm{dl}$, TG/HDL-C $>3$ o colesterol total/HDL-C $>5$ en hombres y $>4,5$ en mujeres), ECG anormal (definido como presencia de: fibrilación auricular, ondas $T$ negativas patológicas, ondas Q patológicas, alteraciones del segmento ST, taquicardia, bloqueo $A V \geq 2$ grado y/o bloqueo de rama izquierda), PEG anormal (definido como presencia de alteraciones ST-T, arritmia ventricular, o angor asociados al esfuerzo), hipertrofia del ventrículo izquierdo (definido por ecocardiograma como septum interventricular $\geq 12 \mathrm{~mm}$ o pared posterior $\geq 11 \mathrm{~mm}$ ), dilatación del ventrículo izquierdo (definido como diámetro de fin de diástole $\geq 55 \mathrm{~mm}$ ), dilatación aórtica (definido como raíz aórtica $>37 \mathrm{~mm}$ en el eje paraesternal largo), presencia de anomalías cardíacas (válvula aórtica bicúspide), radiografía de tórax anormal (presencia de nódulos o neumotórax), o de esteatosis hepática (definida por ecografía abdominal).

También se definieron por consenso y criterio médico la presencia de ECP que requiriera estudios adicionales y/o terapéutica.

Se estimó el riesgo cardiovascular a 10 años utilizando las ecuaciones de riesgo recomendadas en las últimas guías de estimación de riesgo y de colesterol de la American Heart Association/American College of Cardio$\log y$, del año 2013. Utilizando estas ecuaciones (pooled cohort risk equations) se categorizaron los pacientes como de bajo riesgo $(<5 \%)$, riesgo intermedio $(5-7,4 \%)$ y riesgo elevado $(\geq 7,5 \%)^{10,11}$.

Finalmente se discriminó a los pacientes según el índice de masa corporal (IMC), en bajo $\left(<18,5 \mathrm{~kg} / \mathrm{m}^{2}\right)$, saludable $\left(18,5-24,9 \mathrm{~kg} / \mathrm{m}^{2}\right)$, sobrepeso $\left(25-29,9 \mathrm{~kg} / \mathrm{m}^{2}\right)$ u obesidad $\left(\geq 30 \mathrm{~kg} / \mathrm{m}^{2}\right)$.

Todos los procedimientos fueron realizados acorde a los estándares de ética del comité de investigación institucional, y en cumplimiento con la declaración de Helsinki de 1964 y las adendas posteriores. Se obtuvo el consentimiento informado escrito de todos los individuos incluidos en el estudio para la difusión de la información (habeas data).

\section{Análisis estadístico}

Las variables categóricas se expresaron como cuentas y porcentajes y las variables continuas como media \pm desviación estándar.

Las comparaciones entre grupos se realizaron mediante pruebas de Student (de muestras independientes) en caso de variables continuas, y pruebas de chi cuadrado en variables discretas. Las correlaciones entre variables 
TABLA 1. Demografía.

\begin{tabular}{|c|c|c|c|c|}
\hline & Total $(n=311)$ & Mujeres $(n=149)$ & Hombres $(n=162)$ & $p$ \\
\hline Edad (años $\pm D E)$ & $42,4 \pm 14,8$ & $43,6 \pm 14,6$ & $41,3 \pm 15,0$ & 0,17 \\
\hline Diabetes (\%) & $9(3 \%)$ & $7(5 \%)$ & $2(1 \%)$ & 0,09 \\
\hline Tabaquismo (\%) & $57(18 \%)$ & $24(16 \%)$ & $33(20 \%)$ & 0,38 \\
\hline Dislipemia (\%) & $46(15 \%)$ & $20(13 \%)$ & $26(16 \%)$ & 0,53 \\
\hline Hipertensión (\%) & $34(11 \%)$ & $14(9 \%)$ & $20(12 \%)$ & 0,47 \\
\hline Hipotiroidismo (\%) & $35(11 \%)$ & $25(17 \%)$ & $10(6 \%)$ & 0,004 \\
\hline Antecedentes oncológicos (\%) & $9(3 \%)$ & $6(4 \%)$ & $3(2 \%)$ & 0,32 \\
\hline Actividad física regular (\%) & $193(62 \%)$ & $86(58 \%)$ & $107(66 \%)$ & 0,16 \\
\hline Frecuencia cardíaca (lpm) & $75,3 \pm 10,3$ & $75,1 \pm 10,6$ & $75,5 \pm 10,0$ & 0,79 \\
\hline $\mathrm{PAS}(\mathrm{mmHg} \pm \mathrm{DE})$ & $115,0 \pm 14,7$ & $110,4 \pm 13,4$ & $119,2 \pm 14,6$ & $<0,0001$ \\
\hline PAD (mmHg $\pm D E)$ & $71,9 \pm 10,5$ & $69,3 \pm 9,6$ & $74,3 \pm 10,8$ & $<0,0001$ \\
\hline PAS $\geq 140 \mathrm{mmHg}$ y/o PAD $\geq 90 \mathrm{mmHg}(\%)$ & $32(12 \%)$ & $7(6 \%)$ & $25(18 \%)$ & 0,002 \\
\hline Hipertensión (antecedente y/o medida) & $55(18 \%)$ & $19(13 \%)$ & $36(22 \%)$ & 0,04 \\
\hline
\end{tabular}

PAS: presión arterial sistólica. PAD; presión arterial diastólica.

TABLA 2. Indice de masa corporal (IMC).

\begin{tabular}{|c|c|c|c|c|}
\hline & Total & Mujeres & Hombres & $p$ \\
\hline Índice de masa corporal (kg/m2士DE) & $25,2 \pm 4,1$ & $24,1 \pm 4,1$ & $26,3 \pm 3,9$ & 0,0001 \\
\hline Bajo $\left(\mathrm{IMC}<18,5 \mathrm{~kg} / \mathrm{m}^{2}\right)$ & $6(2 \%)$ & $4(3 \%)$ & $2(1 \%)$ & $<0,0001^{*}$ \\
\hline Saludable (IMC 18,5-24,9 kg/m²) & $156(50 \%)$ & $96(64 \%)$ & $60(37 \%)$ & \\
\hline Sobrepeso (IMC 25-29,9 kg/m²) & $100(32 \%)$ & $28(19 \%)$ & $72(44 \%)$ & \\
\hline Obesidad $\left(\mathrm{IMC} \geq 30 \mathrm{~kg} / \mathrm{m}^{2}\right)$ & $49(16 \%)$ & $21(14 \%)$ & $28(17 \%)$ & \\
\hline
\end{tabular}

* chi cuadrado.

TABLA 3. Riesgo de eventos de 10 años según las ecuaciones de riesgo de la American Heart Association/American College of Cardiology.

\begin{tabular}{|c|c|c|c|c|c|}
\hline & $\begin{array}{c}\text { Total } \\
(n=311)\end{array}$ & $\begin{array}{c}\text { Hombres } \geq 40 \\
(n=93)\end{array}$ & $\begin{array}{c}\text { Hombres }<40 \\
(n=69)\end{array}$ & $\begin{array}{c}\text { Mujeres } \geq 40 \\
(n=88)\end{array}$ & $\begin{array}{c}\text { Mujeres }<40 \\
(n=61)\end{array}$ \\
\hline Riesgo & $3,6 \pm 5,6$ & $7,4 \pm 7,2$ & $1,1 \pm 0,9$ & $3,6 \pm 5,5$ & $0,7 \pm 0,6$ \\
\hline Riesgo $<5 \%$ & $249(80 \%)$ & $45(50 \%)$ & $130(100 \%)$ & $74(82 \%)$ & $62(100 \%)$ \\
\hline $5-7,4 \%$ & $19(6 \%)$ & $15(17 \%)$ & $0(0 \%)$ & $4(4 \%)$ & $0(0 \%)$ \\
\hline$\geq 7,5 \%$ & $43(14 \%)$ & $31(34 \%)$ & $0(0 \%)$ & $12(13 \%)$ & $0(0 \%)$ \\
\hline Chequeo anormal & $173(56 \%)$ & $72(77 \%)$ & $34(49 \%)$ & $42(48 \%)$ & $25(41 \%)$ \\
\hline Estudios adicionales & $57(18 \%)$ & $24(26 \%)$ & $6(9 \%)$ & $16(18 \%)$ & $11(18 \%)$ \\
\hline Terapéutica & $72(23 \%)$ & $36(39 \%)$ & $10(15 \%)$ & $18(21 \%)$ & $8(13 \%)$ \\
\hline
\end{tabular}

continuas se realizaron utilizando coeficientes de correlación de Pearson. El nivel de significación estadística se estableció en un valor de p menor a 0,05. Los análisis se realizaron con el software de estadística SPSS, versión 22.0 (Chicago, Illinois, EE.UU.).

\section{RESULTADOS}

Durante el mes de marzo de 2015, se reclutaron 311 individuos asintomáticos que concurrieron a nuestra institución a realizarse un ECP. La edad media fue de

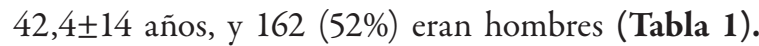
Del total de los pacientes incluidos, 193 (62\%) realizaban actividad física de forma regular, sin diferencias significativas entre sexos. Cincuenta y siete (18\%) pacientes eran fumadores, no encontrándose diferencias significativas entre sexos. Del total de los sujetos reclutados, 9 (3\%) eran diabéticos, 46 (15\%) tenían dislipemia, 34 (11\%) hipertensión, sin diferencias entre sexos. La prevalencia de hipotiroidismo fue mayor en mujeres que en hombres ( $17 \%$ vs. $6 \% ; \mathrm{p}=0,004)$, mientras que los hombres presentaron mayor IMC que las mujeres $\left(26,3 \pm 3,9 \mathrm{~kg} / \mathrm{m}^{2}\right.$ vs. $\left.24,1 \pm 4,1 \mathrm{~kg} / \mathrm{m}^{2} ; \mathrm{p}<0,0001\right)$.
Apenas el 50\% de los pacientes presentaron un IMC saludable, en su mayor medida mujeres. Se observaron diferencias significativas en cuanto a la categorización del IMC, en particular, 100 (62\%) hombres presentaron sobrepeso u obesidad (Tabla 2), y 72 (78\%) de los hombres mayores 40 años.

Treinta y dos (10\%) pacientes tenían al momento de la consulta una presión arterial sistólica $\geq 140 \mathrm{mmHg}$ y/o diastólica $\geq 90 \mathrm{mmHg}$, de los cuales 21 (65\%) no tenían antecedente de hipertensión.

\section{Perfil de riesgo y frecuencia de ECP anormales}

La mayor parte $(249 / 311,80 \%)$ de los pacientes se ubicó dentro del grupo de bajo riesgo de eventos $(<5 \%)$, observándose un total de 43 (14\%) pacientes dentro del grupo de riesgo elevado de eventos ( $\geq 7,5 \%)$. Los pacientes de riesgo intermedio y elevado se agruparon en mayor medida dentro de los hombres mayores de 40 años, con prevalencia de $17 \%$ y $34 \%$, respectivamente (Tabla 3 ). El 56\% (173/311) de los ECP fueron clasificados como anormales, concentrándose la mayor proporción en hombres mayores de 40 años (Tabla 3). Las causas más frecuentes fueron la presencia de un perfil lipídi- 
TABLA 4. Glucemia y perfil lipídico.

\begin{tabular}{|c|c|c|c|c|}
\hline & Total & Mujeres & Hombres & $p$ \\
\hline Glucemia (mg/dl) & $91,7 \pm 13,3$ & $90,0 \pm 13,3$ & $93,3 \pm 13,2$ & 0,03 \\
\hline Glucemia $\geq 100$ & $44(14 \%)$ & $16(11 \%)$ & $28(17 \%)$ & 0,11 \\
\hline Colesterol anormal* & $80(26 \%)$ & $21(14 \%)$ & $59(37 \%)$ & $<0,0001$ \\
\hline Colesterol total (mg/dl) & $197,7 \pm 37,3$ & $202,0 \pm 35,3$ & $193,9 \pm 38,8$ & 0,055 \\
\hline Col total >200 & $143(46 \%)$ & $73(49 \%)$ & $70(43 \%)$ & 0,36 \\
\hline Colesterol HDL (mg/l) & $54,0 \pm 24,0$ & $62,4 \pm 31,2$ & $46,3 \pm 9,5$ & $<0,0001$ \\
\hline Índice aterogénico elevado† & $70(23 \%)$ & $12(8 \%)$ & $58(36 \%)$ & $<0,0001$ \\
\hline Triglicéridos (mg/dl) & $102,5 \pm 67,5$ & $91,5 \pm 52,6$ & $112,6 \pm 75,6$ & 0,006 \\
\hline$<150$ & $262(84 \%)$ & $132(89 \%)$ & $130(80 \%)$ & $0,05 \ddagger$ \\
\hline 150-199 & $25(8 \%)$ & $11(7 \%)$ & $14(9 \%)$ & \\
\hline$\geq 200$ & $24(8 \%)$ & $6(4 \%)$ & $18(11 \%)$ & \\
\hline TG/HDL-C ratio & $2,1 \pm 1,6$ & $1,7 \pm 1,4$ & $2,6 \pm 1,7$ & $<0,0001$ \\
\hline $\mathrm{TG} / \mathrm{HDL}-\mathrm{C}>3$ & $64(21 \%)$ & $15(10 \%)$ & $49(30 \%)$ & $<0,0001$ \\
\hline Colesterol LDL (mg/dl) & $124,6 \pm 31,9$ & $123,8 \pm 29,5$ & $125,2 \pm 34,0$ & 0,70 \\
\hline$<100$ & $70(23 \%)$ & $32(22 \%)$ & $38(24 \%)$ & $0,90 \neq$ \\
\hline $100-129$ & $107(35 \%)$ & $53(36 \%)$ & $54(34 \%)$ & \\
\hline $130-160$ & $101(33 \%)$ & $50(34 \%)$ & $51(32 \%)$ & \\
\hline$>160$ & $32(10 \%)$ & $14(9 \%)$ & $18(11 \%)$ & $>160$ \\
\hline
\end{tabular}

t: colesterol total/HDL $>5$ en hombres $y>4,5$ en mujeres. $\neq$ : chi cuadrado.

TABLA 5. Estudios cardiológicos.

\begin{tabular}{|c|c|c|c|c|}
\hline & Total & Mujeres & Hombres & $p$ \\
\hline \multicolumn{5}{|l|}{ ECG y ergometría (PEG) } \\
\hline ECG anormal & $10(3 \%)$ & $6(4 \%)$ & $4(3 \%)$ & 0,53 \\
\hline PEG anormal & $17(6 \%)$ & $7(5 \%)$ & $10(6 \%)$ & 0,63 \\
\hline PEG anormal por infradesnivel ST & $10(3 \%)$ & $4(3 \%)$ & $6(4 \%)$ & 0,75 \\
\hline METS $<7$ & $46(15 \%)$ & $22(15 \%)$ & $24(16 \%)$ & 1,0 \\
\hline \multicolumn{5}{|l|}{ Ecocardiograma } \\
\hline Hipertrofia VI & $26(8 \%)$ & $5(3 \%)$ & $21(13 \%)$ & 0,003 \\
\hline Dilatación VI & $5(2 \%)$ & 0 & $5(3 \%)$ & 0,06 \\
\hline Dilatación Al & $21(7 \%)$ & $4(3 \%)$ & $17(11 \%)$ & 0,006 \\
\hline Dilatación raíz aórtica & $8(3 \%)$ & $1(1 \%)$ & $7(4 \%)$ & 0,07 \\
\hline Insuficiencia mitral & $49(16 \%)$ & $31(21 \%)$ & $18(11 \%)$ & 0,03 \\
\hline Insuficiencia aórtica & $13(4 \%)$ & $6(4 \%)$ & $7(4 \%)$ & 1,0 \\
\hline Disfunción diastólica & $11(4 \%)$ & $5(3 \%)$ & $6(4 \%)$ & 1,0 \\
\hline Aorta bicúspide & $2(1 \%)$ & $1(1 \%)$ & $1(1 \%)$ & 1,0 \\
\hline
\end{tabular}

co anormal, anemia y, en menor medida, alteraciones del ECG o del ecocardiograma. Sin embargo, dichos hallazgos fueron considerados clínicamente relevantes (con potencial solicitud de estudios adicionales y/o necesidad de terapéutica) en aproximadamente el $20 \%$ de los casos (Tabla 3).

\section{Análisis de sangre}

Con respecto a variables relevantes de laboratorio, 44 (14\%) pacientes presentaron glucemia mayor de 100 $\mathrm{mg} / \mathrm{dl}$ y 146 (46\%), un colesterol total mayor a 200 $\mathrm{mg} / \mathrm{dl}$, sin diferencias significativas entre hombres y mujeres (Tabla 4).

Los niveles de HDL-C fueron menores en hombres que en mujeres $(46,3 \pm 9,5 \mathrm{mg} / \mathrm{dl}$ vs. $62,4 \pm 31,2 \mathrm{ml} / \mathrm{dl}$; $\mathrm{p}<0,0001)$. Cincuenta y ocho $(36 \%)$ hombres presentaron un índice aterogénico elevado, comparado con $12(8 \%)$ mujeres $(\mathrm{p}<0,0001)$. Del mismo modo, 49 (30\%) hombres presentaron un TG/HDL-C elevado (marcador subrogante de resistencia a la insulina), comparado con $15(10 \%)$ mujeres $(\mathrm{p}<0,0001)$. Con res- pecto al colesterol LDL, 133 (43\%) pacientes presentaron niveles mayores a $130 \mathrm{mg} / \mathrm{dl}$, sin diferencias entre sexos (Tabla 4).

\section{Estudios diagnósticos}

Se identificaron alteraciones electrocardiográficas relevantes en 10 (3\%) pacientes (incluyendo alteraciones de la repolarización, extrasístoles ventriculares y trastornos de conducción). La prueba ergométrica resultó anormal por infradesnivel del segmento ST en 10 (3\%) pacientes. Cuarenta y seis (15\%) pacientes presentaron una capacidad funcional limitada (<7 METS), sin diferencias entre sexos.

En cuanto al análisis ecocardiográfico, 26 (8\%) pacientes presentaron hipertrofia del ventrículo izquierdo, siendo más prevalente en varones $[21(13 \%)$ vs. 5 (3\%); $\mathrm{p}=0,003]$. De estos pacientes, 7 (27\%) presentaron como antecedente clínico hipertensión y en otros 7 (27\%) se registró hipertensión no conocida.

Cinco pacientes (2\%) presentaron algún grado de dilatación del ventrículo izquierdo, 21 (7\%) presentaron 
dilatación de la aurícula izquierda (área mayor de 21 $\mathrm{cm}^{2}$ ), y 8 (3\%) presentaron dilatación de la raíz aórtica. Con respecto a los parámetros funcionales cardíacos, se observó algún grado de insuficiencia mitral o aórtica en 49 (16\%) y 13 (4\%) pacientes, respectivamente, con mayor prevalencia de la primera en mujeres $(\mathrm{p}=0,03)$. En relación al perfil metabólico, la ecografía de abdomen, realizada solo en mayores de 50 años $(n=102)$, evidenció esteatosis hepática en $9(9 \%)$ pacientes [7 (14\%) hombres y 2 (4\%) mujeres; $\mathrm{p}=0,06]$.

De los pacientes en que se realizó una radiografía de tórax $(n=208), 9$ (4\%) fueron clasificadas como patológicas debido a la presencia de opacidades nodulares o neumotórax, sin diferencias entre sexos $(\mathrm{p}=0,91)$.

\section{Correlaciones}

Si bien muy débiles, se evidenciaron correlaciones significativas entre la edad y el IMC $(r=0,32 ; p<0,0001)$, la glucemia $(r=0,34 ; p<0,0001)$, el colesterol total $(r=0,35 ; p<0,0001)$, el LDL-C $(r=0,31 ; p<0,0001)$, el área auricular izquierda $(\mathrm{r}=0,24, \mathrm{p}<0,0001)$, y el diámetro de fin de diástole del VI $(\mathrm{r}=-0,15 ; \mathrm{p}=0,01)$. No se observó relación entre la edad y el HDL-C $(\mathrm{r}=0,04 ; \mathrm{p}=0,46)$, ni el espesor del septum interventricular $(\mathrm{r}=0,10 ; \mathrm{p}=0,08)$. También se observaron correlaciones significativas (si bien débiles) entre el riesgo a 10 años y variables no incluidas en dicha estimación, como el IMC ( $r=0,34 ; p<0,0001)$, los niveles de triglicéridos $(r=0,28, p<0,0001)$, el área auricular izquier$\mathrm{da}(\mathrm{r}=0,28 ; \mathrm{p}<0,0001)$ y la presión arterial diastólica $(\mathrm{r}=0,27, \mathrm{p}<0,0001)$.

\section{DISCUSIÓN}

Los principales hallazgos de nuestro estudio se pueden resumir de la siguiente forma: 1) un porcentaje relativamente elevado de hombres sanos mayores de 40 años presentaron un perfil de riesgo elevado; 2) más de la mitad de los hombres presentaron sobrepeso u obesidad (78\% de los mayores de 40 años); 3 ) el 37\% de los hombres ( $48 \%$ de los mayores de 40 años) presentaron un perfil lipídico anormal; y 4) se identificaron hallazgos clínicamente relevantes en aproximadamente el 20\% de los ECP.

El perfil cardiometabólico adverso observado en nuestro estudio que involucró ECP de pacientes sanos evidencia el deficiente control de FRC en la población estudiada. Es notorio y preocupante que dicha aparente falla en las estrategias de prevención se ponga en evidencia en una población relativamente joven (edad media 42 años) en pleno desempeño laboral y dentro de un estrato socioeconómico elevado. Cabe destacar que estas estadísticas se dan a pesar de que el 60\% de los pacientes refirió realizar actividad física de forma habitual.

En ese contexto, apenas la mitad de los pacientes presentaron un IMC saludable, mientras que, en cuanto a las cifras de presión arterial, es destacable que el 65\% de los pacientes con hipertensión lo desconocían.

\section{Perfil lipídico y glucémico}

El estudio multinacional INTERHEART, que incluyó pacientes de 52 países, demostró que las alteraciones del metabolismo lipoproteico son el principal factor para el desarrollo de infarto de miocardio ${ }^{12}$.

En nuestro estudio, a pesar de que solo el $15 \%$ de los pacientes tenían antecedentes de hipercolesterolemia, se identificaron cifras significativamente más elevadas en cuanto a la prevalencia de alteraciones en el perfil lipídico (26\%), incluyendo casi el 50\% de los hombres mayores de 40 años. De hecho, el índice aterogénico de Castelli, un excelente predictor de riesgo cardiovascular, se encontró elevado en el $23 \%$ de los casos ( $43 \%$ de los hombres mayores de 40 años $)^{13}$.

La American Diabetes Association define como glucemia anormal en ayunas, también conocida como prediabetes, a la presencia de glucemia $\geq 100 \mathrm{mg} / \mathrm{dl}^{14}$. Este estado prediabético se ha asociado a la resistencia a la insulina y a un incremento del riesgo de desarrollo de diabetes tipo II. El 14\% de los pacientes incluidos en nuestro estudio presentó prediabetes, incluyendo el $28 \%$ de los hombres mayores de 40 años.

En la misma línea, evidenciamos una elevada prevalencia de un marcador subrogante de resistencia a la insulina (razón TG/HDL-C) particularmente en hombres (30\% del total y $40 \%$ en mayores de 40 años $)^{15}$.

\section{Estudios diagnósticos}

En términos generales, si bien el 56\% de los ECP fueron considerados anormales debido a la presencia de algún tipo de alteración (predominantemente del análisis de sangre), solo en el $20 \%$ se identificaron hallazgos clínicamente relevantes.

Con respecto a los ECG y a la prueba ergométrica, solo el 3\% de los pacientes presentó alteraciones. Cabe destacar que, llamativamente, el 15\% de los pacientes presentó una capacidad funcional limitada (<7 METS). Numerosos estudios han descrito la asociación entre los niveles de aptitud física y el riesgo de la mortalidad por enfermedad cardiovascular, incluyendo el Cooper Center Longitudinal Study, que demostró que la presencia de un único registro de baja aptitud física se asoció a un incremento significativo de la mortalidad 30 años más tarde ${ }^{16}$. Otros grandes estudios e incluso un reciente metaanálisis han demostrado de forma consistente una relación inversa entre la condición física y la mortalidad en personas con y sin enfermedad coronaria establecida, independientemente de los $\mathrm{FRC}^{17-19}$.

En su estudio, Berry et al. reportaron que en un hombre de 55 años de edad con una muy baja aptitud física (6 METS), el riesgo de morir por enfermedad cardiovascular es de casi un 30\% a 30 años; mientras que un hombre capaz de realizar una prue- 
ba ergométrica de 10 METS tendría un riesgo de muerte por enfermedad cardiovascular de sólo $10 \%$ a 30 años ${ }^{16}$.

En algunos medios o sociedades se ha propuesto la inclusión de la ecocardiografía en los protocolos de tamizaje de enfermedades cardiovasculares para grandes grupos poblacionales; la ecocardiografía es una herramienta de gran utilidad para el diagnóstico de miocardiopatías y para la evaluación de la patología aórti$\mathrm{ca}^{20}$. En nuestro estudio, el $8 \%$ de los pacientes presentó hipertrofia del VI (16\% de los hombres mayores de 40 años), mientras que otros hallazgos de escasa relevancia se encontraron con menor frecuencia.

En el contexto de la valoración del perfil cardiometabólico, se debe destacar la presencia de esteatosis hepática en el $9 \%$ de los pacientes mayores de 50 años. Existe extensa evidencia que vincula el hígado graso de forma independiente no solo con una mayor prevalencia de resistencia a la insulina y diabetes, sino también con la presencia de inflamación y aterosclerosis subclínica $^{21-23}$.

\section{Implicancias clínicas e impacto del perfil cardiometabólico adverso}

$\mathrm{Si}$ bien nuestro estudio presenta las limitaciones propias de todo estudio epidemiológico, el presente registro permite estimar el perfil de FRC en el "mundo real" de individuos sanos dentro del estrato socioeconómico medio-elevado de la Ciudad Autónoma de Buenos Aires.

El hallazgo de una gran cantidad de variables alteradas en torno al perfil cardiometabólico dentro de una población joven, sana, activa y a priori de muy bajo riesgo podría eventualmente atribuirse a la existencia de una conciencia colectiva de que el control periódico mediante el ECP reemplazaría la importancia del estilo de vida y control de factores de riesgo.

En nuestro medio, la ENASE (Encuesta NAcional de SEguimiento sobre factores de riesgo cardiovascular) demostró que un plan de educación y entrenamiento de los médicos enfocado para actuar sobre medidas higiénico-dietéticas y sobre los hábitos de actividad física produce beneficios significativos en la salud cardiovascular. En dicho estudio, Zilberman et al. demostraron que dicho entrenamiento conduce a mejorar significativamente el perfil lipídico, la presión arterial, y los índices antropométricos ${ }^{24}$.

El impacto de las estrategias de prevención se pone de manifiesto también por las estadísticas de mortalidad cardiovascular en los Estados Unidos entre los años 1980 y 2000 , donde aproximadamente la mitad de la reducción de la mortalidad se atribuyó a la reducción de los $\mathrm{FRC}^{2}$. Otro estudio que ofrece prueba contundente acerca de ello es el INTERHEART, que demostró que los FRC modificables (incluyendo perfil lipídico adverso, tabaquismo, hipertensión, diabetes, obesi$\mathrm{dad}$, factores psicosociales, dieta, y los patrones de ac- tividad física) son responsables de la mayor parte del riesgo de infarto independientemente de la edad y la región ${ }^{12}$.

De no iniciarse estrategias y/o campañas de prevención en poblaciones como la de nuestro estudio, es de preverse que conduzca a mediano o largo plazo a un impacto no solo desde el punto de vista clínico sino también económico para los sistemas de salud.

Según las últimas guías de la American Heart Association/American College of Cardiology acerca de la estimación de riesgo y del manejo del colesterol, los pacientes con riesgo $\geq 7,5 \%$ tienen indicación (clase I) de dosis elevadas de estatinas, mientras que en aquellos con riesgo $\geq 5 \%$ sería razonable (IIa) la indicación de dosis moderadas de estatinas. Si bien han recibido numerosas críticas y es por tanto debatible su utilidad, según estas guías el $14 \%$ de los pacientes estudiados tendrían indicación de dosis elevadas de estatinas, incluyendo el 34\% de los hombres mayores de 40 años, y un total del 51\% de los hombres mayores de 40 años con indicación de estatinas de clase $\geq \mathrm{IIa}^{25}$.

\section{Limitaciones}

Si bien presenta las limitaciones propias de todo estudio epidemiológico, el presente registro permite estimar el perfil de FRC en el "mundo real" de individuos sanos dentro del fragmento socio-económico medio-alto de la Ciudad Autónoma de Buenos Aires. No se adquirieron de rutina las medidas de perímetro abdominal, no permitiendo la estimación de la prevalencia de síndrome metabólico en esta muestra. Además, las ecuaciones de riesgo incluidas no se encuentran validadas para menores de 40 años y la determinación de la presencia o ausencia de ECP normal o anormal se basó en un criterio arbitrario, si bien consensuado entre el equipo médico involucrado. Finalmente, se debe destacar que nuestro estudio no pone a juicio la utilidad ni el valor pronóstico de implementar ECP, ni tiene el propósito de fomentar su universalización en nuestro medio. Por el contrario, se limita a reportar los hallazgos de una práctica implementada por diversos sistemas prepagos en la Argentina.

\section{CONCLUSIONES}

En este registro de exámenes clínicos periódicos de salud, identificamos tasas relativamente elevadas de perfil cardiometabólico adverso en una población mayormente joven, socialmente activa, con manejo subóptimo de los factores de riesgo coronario. Esto ofrece la oportunidad de incorporar herramientas de concientización y reforzar medidas de prevención con el objetivo de evitar, de persistir dicho perfil desfavorable, un potencial impacto tanto a nivel clínico como económico. 


\section{BIBLIOGRAFÍA}

1. Lozano R, Naghavi M, Foreman K, et al. Global and regional mortality from 235 causes of death for 20 age groups in 1990 and 2010: a systematic analysis for the Global Burden of Disease Study 2010. Lancet 2012; 380(9859):2095-128.

2. Ford ES, AjaniUA, Croft JB, et al. Explaining the decrease in U.S. deaths from coronary disease, 1980-2000. New Engl J Med 2007;356(23):2388-98.

3. Rodríguez-Granillo GA, Rosales MA, MadeoM, DiezE, RodríguezAE. Factores de riesgo y extensión de la enfermedad coronaria evaluada por angiografía coronaria no invasiva. Rev Argent Cardiol 2008;76:112-7.

4. Chiuve SE, Fung TT, Rexrode KM, et al. Adherence to a low-risk, healthy lifestyle and risk of sudden cardiac death among women. JAMA 2011;306(1):62-9.

5. Ford ES, Greenlund KJ, Hong Y. Ideal cardiovascular health and mortality from all causes and diseases of the circulatory system among adults in the United States. Circulation 2012;125(8):987-95.

6. Ahmed HM, Blaha MJ, Nasir K, et al. Low-risk lifestyle, coronary calcium, cardiovascular events, and mortality: results from MESA. Am J Epidemiol 2013;178(1):12-21.

7. Shaw LJ, Giambrone AE, Blaha MJ, et al. Long-term prognosis after coronary artery calcification testing in asymptomatic patients: a cohortstudy. Ann Intern Med 2015;163(1):14-21.

8. Hartiala O, Magnussen CG, Kajander S, et al. Adolescence risk factors are predictive of coronary artery calcification at middle age: the cardiovascular risk in young Finns study. J Am Coll Cardiol 2012;60(15):1364-70.

9. PletcherMJ, Bibbins-Domingo K, LiuK, et al. Nonoptimal lipids commonly present in young adults and coronary calcium later in life: the CARDIA (Coronary Artery Risk Development in Young Adults) study. Ann Intern Med 2010;153(3):137-46.

10. Stone NJ, Robinson JG, Lichtenstein AH, et al. 2013 ACC/AHA guideline on the treatment of blood cholesterol to reduce atherosclerotic cardiovascular risk in adults: a report of the American College of Cardiology/American Heart Association Task Force on Practice Guidelines. J Am Coll Cardiol 2014;63(25 Pt B):2889-934

11. Goff DC, Jr., Lloyd-Jones DM, Bennett G, et al. 2013 ACC/AHA guideline on the assessment of cardiovascular risk: a report of the American College of Cardiology/American Heart Association Task Force on Practice Guidelines. J Am Coll Cardiol 2014;63(25 Pt B):2935-59.

12. Yusuf S, Hawken S, Ounpuu S, et al. Effect of potentially modifiable risk factors associated with myocardial infarction in 52 countries (the INTERHEART study): case-control study. Lancet 2004;364(9438):937-52.
13. ArsenaultBJ, RanaJS, Stroes ES, et al. Beyondlow-density lipoprotein cholesterol: respective contributions of non-high-density lipoprotein cholesterol levels, triglycerides, and the total cholesterol/high-density lipoprotein cholesterol ratio to coronary heart disease risk in apparently healthy men and women. J Am Coll Cardiol 2009;55(1):35-41.

14. Genuth S, Alberti KG, Bennett P, et al. Follow-up report on the diagnosis of diabetes mellitus. Diabetes Care 2003;26(11):3160-7.

15. Armato J, Reaven G, Ruby R. Triglyceride/high-density lipoprotein cholesterol concentration ratio identifies accentuated cardio-metabolic risk. Endocr Pract 2015:1-18

16. Berry JD, Willis B, Gupta S, et al. Lifetime risks for cardiovascular disease mortality by cardiorespiratory fitness levels measured at ages 45, 55, and 65 years in men. The Cooper Center Longitudinal Study. J Am Coll Cardiol 2011;57(15):1604-10.

17. Ekelund LG, HaskellWL, Johnson JL, etal. Physical fitness as a predictor of cardiovascular mortality in asymptomatic north american men. The Lipid Research Clinics Mortality Follow-up Study. New Engl J Med 1988;319(21):1379-84.

18. KodamaS, Saito K, Tanaka S, et al. Cardiorespiratory fitness as a quantitative predictor of all-cause mortality and cardiovascular events in healthy men and women: a meta-analysis. JAMA 2009;301(19):2024-35.

19. Myers J, Prakash M, Froelicher V, Do D, Partington S, Atwood JE. Exercise capacity and mortality among men referred for exercise testing. New Engl J Med 2002;346(11):793-801.

20. Boraita Pérez A, Bano Rodrigo A, Berrazueta Fernández JR, et al. Clinical practice guidelines of the Spanish Society of Cardiology for physical activity in patients with cardiac disease. Rev Esp Cardiol 2000;53(5):684-726.

21. Pacifico L, Nobili V, Anania C, Verdecchia P, Chiesa C. Pediatric nonalcoholic fatty liver disease, metabolic syndrome and cardiovascular risk. World J Gastroenterol 2011;17(26):3082-91.

22. AlRifai M, Silverman MG, NasirK, et al. The association of nonalcoholicfatty liver disease, obesity, and metabolic syndrome, with systemic inflammation and subclinical atherosclerosis: the Multi-Ethnic Study of Atherosclerosis (MESA). Atherosclerosis 2015;239(2):629-33.

23. Pisto P, Santaniemi M, Bloigu R, Ukkola O, Kesaniemi YA. Fatty liver predicts the risk for cardiovascular events in middle-aged population: a population-based cohort study. BMJ Open 2014;4(3):e004973.

24. Zilberman JM CL, Woronko E, Vainstein N, Sczygiel V, Ghigi R, Grippo S, A $V$. Resultados de un estudio multicéntrico, no controlado, de seguimiento sobre factores de riesgo cardiovascular. Rev Argent Cardiol 2012;80:130-6.

25. Hecht HS. The sins of the fathers (and mothers) and the 2013 guidelines. JACC Cardiovasc Imaging 2014;7(7):687-9. 